Journal of Patient-Centered

Volume 4

Issue 4 -- Health Disparities and Inequities: Part

Article 17 I

$11-6-2017$

\title{
Variations in Practice of Apnea Test for Brain Death: Review From a Multihospital Health Care System
}

Jeffrey Stein

Marvi V. Rijhwani

Ernesto Brauer

Follow this and additional works at: https://aah.org/jpcrr

Part of the Circulatory and Respiratory Physiology Commons, Critical Care Commons, Health Services Research Commons, Respiratory System Commons, and the Respiratory Tract Diseases Commons

\section{Recommended Citation}

Stein J, Rijhwani MV, Brauer E. Variations in practice of apnea test for brain death: review from a multihospital health care system. J Patient Cent Res Rev. 2017;4:259.

Published quarterly by Midwest-based health system Advocate Aurora Health and indexed in PubMed Central, the Journal of Patient-Centered Research and Reviews (JPCRR) is an open access, peer-reviewed medical journal focused on disseminating scholarly works devoted to improving patient-centered care practices, health outcomes, and the patient experience. 
Background: Health care systems continuously seek to improve patient care through population-level analysis of clinical quality metrics and patient characteristics to identify disparities in care. Nationally, disparities in colorectal cancer (CRC) screening rates have been identified with lower screening rates reported for patients who are uninsured and/or lower socioeconomic status, African American/ black, Asian, and non-English-speaking Hispanic patients. No agerelated CRC screening rate disparities with associated interventions have been reported.

Purpose: Determine and address CRC screening disparities in care provided to eligible patients $>50$ years old in two primary care residency clinics.

Methods: Retrospective analysis using REAL-G (race, ethnicity, age, preferred language, gender) categories and insurance coverage was completed on a 12-month data set to identify presence of CRC screening disparities. Barriers to CRC screening for largest disparity gap were then identified by clinic staff at two family medicine residency clinics (a third primary care clinic in same zip code and service region were used for nonintervention comparison) using the Institute for Healthcare Improvement fishbone approach. The project team, informed by the literature, then identified and implemented targeted interventions, monitoring progress during a 6-month period. Interventions included provider education with periodic reminders regarding system-approved CRC screening options and a workflowbased intervention. Postintervention analysis was completed using same preintervention approach.

Results: The largest CRC screening disparity for region and clinics was associated with age, with screening gaps ranging from $13 \%$ to $15 \%$ between populations aged $50-54$ years versus $>65$ years. CRC screening rate disparities by race, ethnicity, and gender were less than $10 \%$. Postintervention, one targeted clinic had a $6 \%$ increase in the CRC screening rates in the target population (age: 50-54) while a second targeted clinic had a $1 \%$ increase in screening rates during this period. The comparison primary care residency clinic had a $1 \%$ decline in CRC screening rates. Differences in insurance utilization types for CRC screening rates by clinic were noted. Differences between targeted clinic screening rates were attributed to successful workflow implementation and provider/staff champions.

Conclusion: Analyzing population data at a micro/clinic level using REAL-G categories can inform targeted interventions that aim to reduce health disparity gaps.

\section{Variations in Practice of Apnea Test for Brain Death: Review From a Multihospital Health Care System}

\section{Jeffrey Stein, Marvi V. Rijhwani, Ernesto Brauer \\ Department of Internal Medicine, Aurora Sinai Medical Center; Bellin Health Pulmonary Clinic, Bellin Health Systems}

Background: Ventilation encompasses both active and passive processes. Air is initially drawn into the lungs due to a negative intrathoracic pressure created using the respiratory muscles, most importantly the diaphragm. In contrast, expiration is the passive relaxation of the respiratory muscles. Oxygenation occurs when oxygen diffuses across the alveolar-capillary membrane. The ability to oxygenate without ventilation has been termed apneic diffusion oxygenation or apneic oxygenation. We believe it is crucial to keep alveoli open in order for adequate oxygenation to occur. This can be achieved with the aid of positive end-expiratory pressure (PEEP). We investigated this concept in patients who are brain-dead because they cannot ventilate. The stimulus to breathe originates from chemoreceptors in the brainstem. These cells respond to a decrease in $\mathrm{pH}$ by triggering the body to take a breath. A positive apnea test confirms that the patient has no functioning brainstem.

Purpose: Determine the rate of $\mathrm{pO}_{2}$ and $\mathrm{pCO}_{2}$ changes during different methods of the apnea test and identify variations in practice within Aurora Health Care.

Methods: Data were collected retrospectively on brain-dead patients older than 18 years. Data points pulled from Epic medical records included serial arterial blood gases (ABGs) that were completed during the apnea test and patient demographics. The rate of change in $\mathrm{pCO}_{2}$ and $\mathrm{pO}_{2}$ was evaluated using both Mann-Whitney and two-sample t-tests comparing a PEEP valve group to all other oxygenation methods.

Results: Eight variations of the test were performed, with median starting $\mathrm{CO}_{2}$ for the oxygenation and PEEP group of 43 and 44 $\mathrm{mmHg}$, respectively ( $95 \%$ confidence interval: $26-53, \mathrm{P}=0.6771)$. Oxygenation group had a mean $\mathrm{CO}_{2}$ increase of $2.95 \mathrm{mmHg} /$ minute, whereas the PEEP valve group increased at $4.60 \mathrm{mmHg} /$ minute. No statistical significance was found $(\mathrm{P}=0.0508)$. Neither was there significant difference between the rate of desaturation between the oxygenation and PEEP valve group $(6.53 \mathrm{mmHg}$ vs $2.60 \mathrm{mmHg}$, respectively; $\mathrm{P}=0.5536$ ).

Conclusion: We found no difference in the rate of $\mathrm{CO}_{2}$ increase comparing the oxygenation group to the PEEP valve group. This suggests that there is no significant component of $\mathrm{CO}_{2}$ washout in the lungs using the PEEP valve setup. A superior method of apneic oxygen was not able to be demonstrated with our results due to an insufficient sample size and practice variations. The most common method to perform the apnea test at our institutions is preoxygenation. Effects of Revision Surgery on Grade of Adverse Local
Tissue Reaction Following Recall of a Modular Hip Implant

\section{Joseph F. Davies, Danielle M. Greer, Susan Truchan}

Aurora Advanced Orthopedics; Center for Urban Population Health; Aurora University of Wisconsin Medical Group; Aurora Research Institute

Background: The Stryker Rejuvenate modular hip implant device allows for greater versatility in matching a patient's anatomy than conventional implants. Device recalls and in vivo metal hypersensitivity after total hip arthroplasty (THA) are common. However, recall of the Rejuvenate implant represents one of the largest recall volumes to date, highlighting our uncertainty regarding causes of device metal fretting/corrosion and adverse local tissue reaction (ALTR). While devices with metal-on-metal bearings historically were culprits for release of metal debris, more recently developed modular-neck devices add opportunity for adjacent metal components to rub together. With the Rejuvenate device, corrosion or fretting likely occurs at the cobalt-chromium neck and titanium stem interface. Cobalt $(\mathrm{Co})$ and chromium $(\mathrm{Cr})$ particles then irritate tissues locally and cause a gradation of problems or indications of ALTR.

Purpose: Quantify the effect of revision surgery on ALTR grade in patients who previously underwent THA receiving the recalled Rejuvenate hip implant.

Methods: We conducted a prospective observational study of all patients who underwent THA performed by a single Aurora Health Care orthopedic surgeon using the Rejuvenate implant. Following implant recall in July 2012, patients were notified via letter/phone and asked to visit regularly (every 3-6 months) for lab work, imaging and device assessment. Using repeated measures multinomial logit analysis we examined the effect of revision surgery on abnormal grade of ALTR (ie, grade 1-4 vs 0), adjusting 